\title{
Influencia del contenido de cromo en el proceso de absorción de nitrógeno por aleaciones de $\mathrm{Fe}-\mathrm{Cr}$
}

Armando Sarmiento Santos*

\section{Resumen}

Recibido:

19 de marzo de 2008

Aceptado:

28 de mayo de 2008
El nitrógeno absorbido por una aleación ferrosa en una descarga luminiscente anormal en atmósfera de nitrógeno puede controlarse a través del tiempo de tratamiento en la descarga y el contenido de cromo de la aleación. Tanto de la cantidad de cromo como de nitrógeno en la aleación depende su microestuctura y sus propiedades mecánicas. En el presente trabajo se someten muestras ferrosas de diferente contenido de cromo a una descarga luminiscente anormal, en atmósfera de nitrógeno, midiéndose la cantidad de nitrógeno absorbido en función del tiempo de tratamiento y el contenido de cromo, observándose y evaluándose el comportamiento del perfil de microdureza en función de dichas variables.

Palabras Claves: Descarga luminiscente anormal, aleaciones de Fe-Cr, Perfil de Microdureza.

\section{Abstract}

Nitrogen absorbed by ferrous alloying in nitrogen abnormal glow discharge can be controlled with the discharge treatment time and the alloy chromium content. Chromium and nitrogen content in the alloy are determinant for its microstructure and its mechanical properties. In this work ferrous alloys with different chromium contents are treated in nitrogen glow discharge. Nitrogen absorbed in the glow is measured as a function of treatment time in the glow and chromium content in the alloy. Then, microhardness profile is observed and evaluated as a function of these variables.

Keywords: Abnormal Glow Discharge, Fe-Cr alloys, Microhardness Profile.
*Universidad Pedagógica y Tecnológica de Colombia FCB-GSEC Escuela de Física Avenida Central del Norte Tunja - Boyacá - Colombia armando.sarmiento@uptc.edu.co 


\section{Introducción}

L a absorción de nitrógeno en todas las fases de una aleación ferrosa, a una temperatura dada, puede mejorarse mediante el aumento de la presión parcial de nitrógeno de la atmósfera gaseosa (Gavriljuk e Berns, 1999), de la misma forma que mediante la adición de un elemento de aleación como el cromo (Zeng et al, 1989; Feichtinger y Zeng, 1991).

El nitrógeno en la aleación puede encontrarse en solución sólida y en la forma de precipitados de nitruros de los diferentes elementos que conforman la aleación. Esto se puede controlar mediante una elección cuidadosa de la temperatura (Wriedt et al, 1987 y Hertzman e Jarl, 1987). A altas temperaturas y bajos tiempos de tratamiento es posible obtener nitrógeno en solución sólida en la red cristalina de aleaciones de $\mathrm{Fe}$-Cr de alto contenido de cromo, además de precipitados de nitruro cúbico de cromo (Sarmiento y Muzart, 2006).

En el presente trabajo se estudia la influencia del contenido de cromo en la cantidad de nitrógeno absorbido en la superficie de aleaciones ferrosas, sometidas a una descarga luminiscente anormal (Chapman, B., 1980) de nitrógeno durante diferentes tiempos de deposición. El perfil de microdureza de las muestras es medido, correlacionándolo con los parámetros de deposición.

\section{Descripción del procedimiento experimental}

Las aleaciones de $\mathrm{Fe}$-Cr fueron fabricadas siguiendo los métodos de la metalurgia de materiales particulados, obteniéndose concentraciones de 13, 18, 23 y $28 \% \mathrm{Cr}$ (Sarmiento, S. A., 2003). Para el proceso de absorción de nitrógeno se utilizó el reactor cuyo esquema se muestra en la Figura 1. Las muestras fueron colocadas en el cátodo de una descarga luminiscente pulsada de $97,5 \mathrm{~N}_{2}+2,5 \mathrm{H}_{2}$ con un flujo de $4 \mathrm{~cm}^{3} / \mathrm{s}$ a una presión de 10 torr. El voltaje de la descarga fue de $700 \mathrm{~V}$ y la temperatura controlada con el ancho del pulso.
1- Cámara del reactor

2- Válvula de vacío

3- Bomba mecánica de dos etapas

4- Medidor de vacío (MKS)

5- Cátodo plano

6- Ánodo cilíndrico

7- Fuente de corriente continua

8. Voltímetro

9. Amperímetro

10. Termopar

11- Registro de temperatura

12- Entrada de gases

13- Válvula de entrada de gases

14- Reguladores de flujo (MKS)

15- Gases

16- Ventana óptica

17- Blindaje térmico
Figura 1 - Esquema del reactor utilizado para tratar las muestras en la descarga luminiscente anormal.

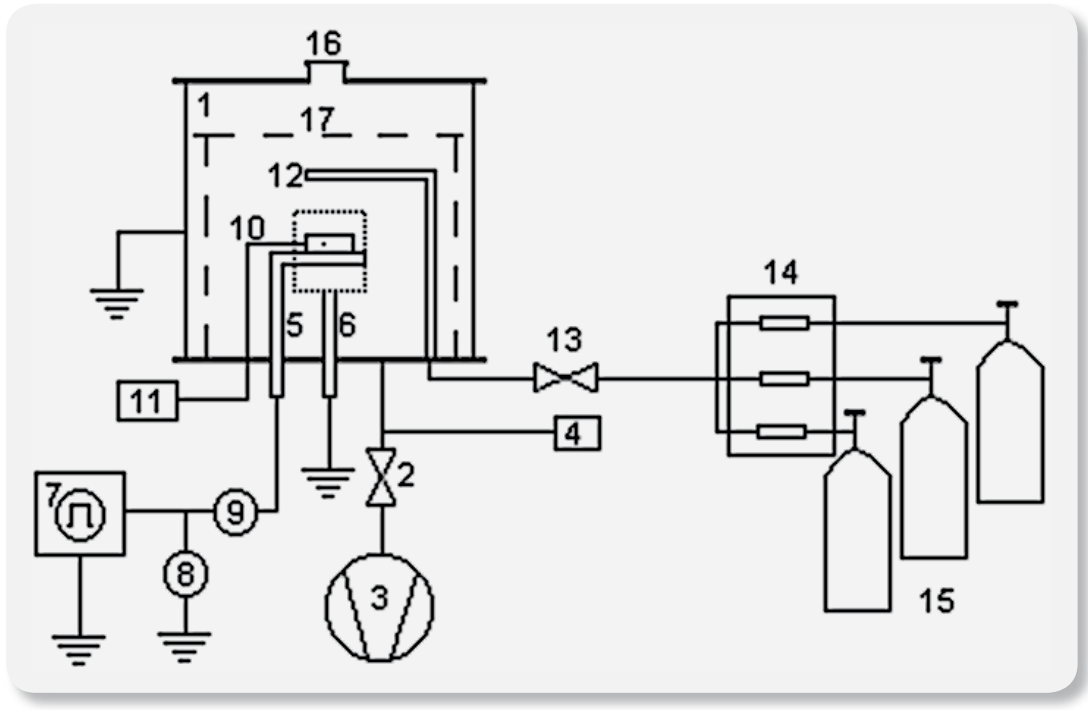


Los tiempos de tratamiento utilizados fueron entre 5 y $120 \mathrm{~min}$. a una temperatura de $950^{\circ} \mathrm{C}$. Las muestras fueron previamente sometidas a una etapa de limpieza durante 15 min. a $900^{\circ} \mathrm{C}$ en una descarga de argón.

En la Figura 2 se representa el ciclo térmico utilizado durante el tratamiento, el cual comprende la etapa de limpieza y la etapa de absorción de nitrógeno.

Figura 2-Ciclo térmico seguido durante el tratamiento de las muestras de Fe-Cr.

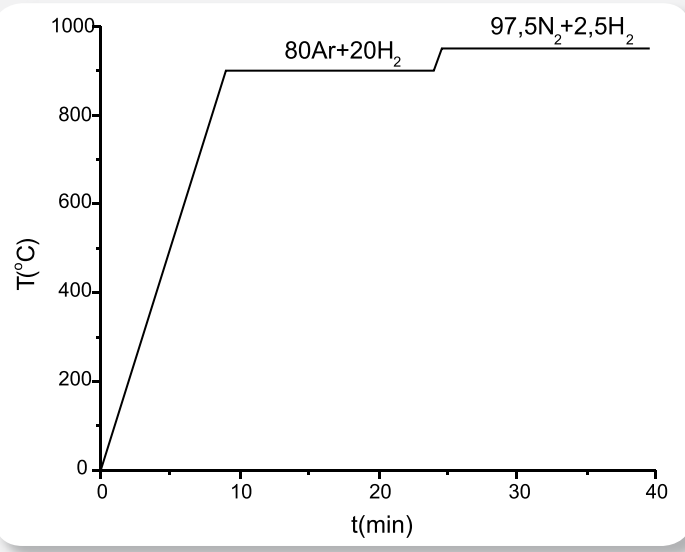

La variación de masa fue medida pesando las muestras antes y después del tratamiento, para ello se utilizó una balanza electrónica de cuatro dígitos. El perfil de microdureza fue realizado con un microdurómetro Shimadzu modelo HMV-2 con una carga de $25 \mathrm{~g}$ y un tiempo de $20 \mathrm{~s}$, después de la preparación metalográfica de las muestras.

\section{Resultados y discusión}

Bajo las condiciones de tratamiento en la descarga luminiscente de nitrógeno $\left(\mathrm{T}=950^{\circ} \mathrm{C}\right.$ y $\mathrm{P}_{\mathrm{N} 2}=97,5 \%$ ), se obtuvo el comportamiento de la variación de masa de las aleaciones de FeCr como función del tiempo de absorción de nitrógeno, ilustrado en la Figura 3(a). La masa de las muestras presenta un aumento rápido para todas las composiciones al comienzo del proceso (tiempos pequeños), debido a la gran demanda de nitrógeno en la superficie de la aleación de Fe-Cr pura. En la medida en que el proceso avanza en el tiempo, el nitrógeno es absorbido y difundido hacia el interior de la muestra, tornando la aleación de Fe-Cr más diluida en la superficie. Así, la demanda de nitrógeno va disminuyendo hasta alcanzarse el equilibrio entre la atmósfera gaseosa y la superficie acorde con las predicciones teóricas (Gavriljuk e Berns, 1999). En éste instante, la superficie de la muestra solamente absorberá la cantidad de nitrógeno suficiente para compensar las pérdidas debidas al proceso de pulverización catódica.

Figura 3- Variación de masa en función del tiempo de absorción de nitrógeno (a) y como función del contenido de cromo (b) en las aleaciones de $\mathrm{Fe}-\mathrm{Cr}$ $\left(\mathrm{T}=950^{\circ} \mathrm{C}, \mathrm{P}_{\mathrm{N} 2}=97,5 \%\right)$.

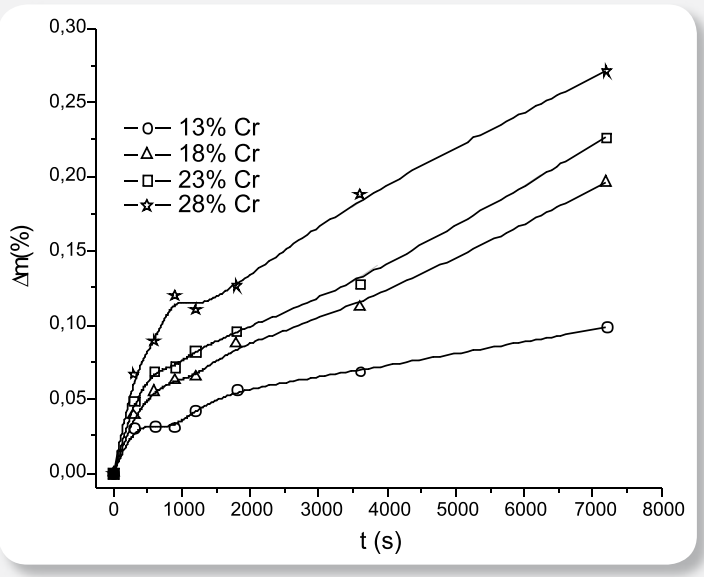

(a)

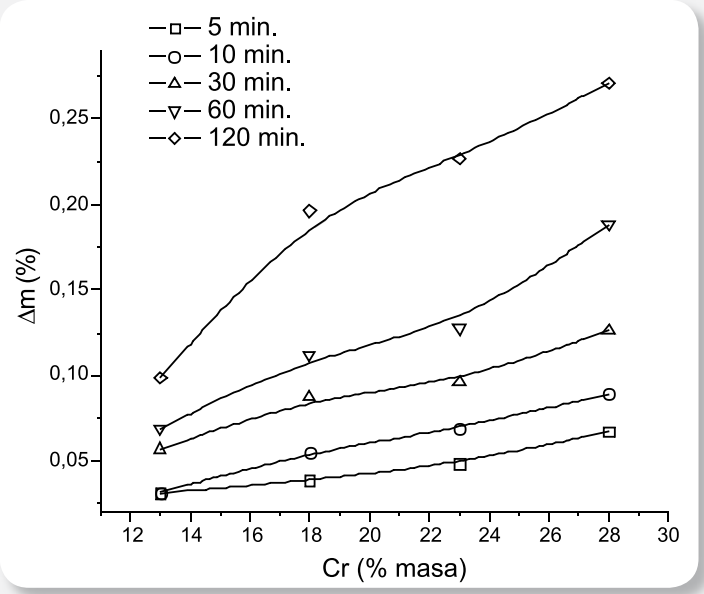

(b) 


\section{Respuestas}

Año 13

No. 1

Junio 2008

ISSN 0122-820X
Influencia del contenido de cromo en el proceso de absorción de nitrógeno por aleaciones de $\mathrm{Fe}-\mathrm{Cr}$.

Figura 4-Perfiles de micro-dureza de las aleaciones de Fe-Cr estudiadas, para 15, 30, 60 y 120 min. de absorción de nitrógeno en la descarga luminiscente anormal a $950^{\circ} \mathrm{C}$.

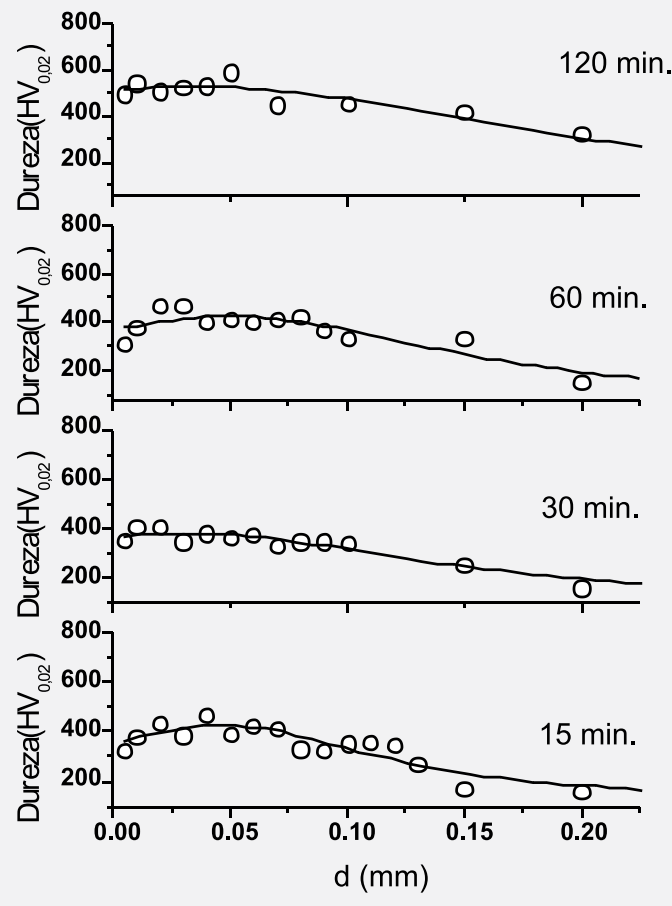

$13 \% \mathrm{Cr}$

16
$120 \mathrm{~min}$.
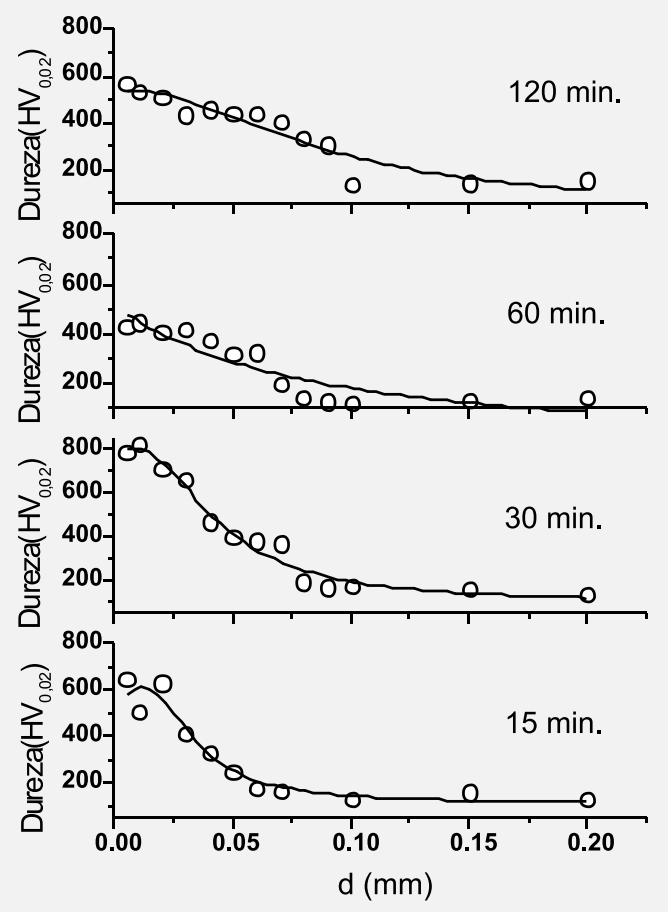

$23 \% \mathrm{Cr}$

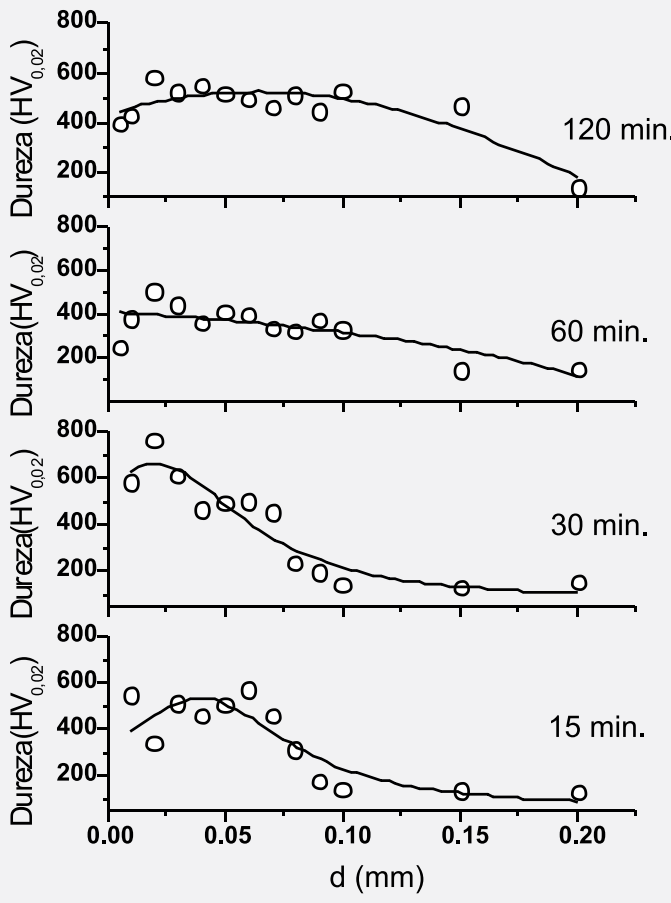

$18 \% \mathrm{Cr}$

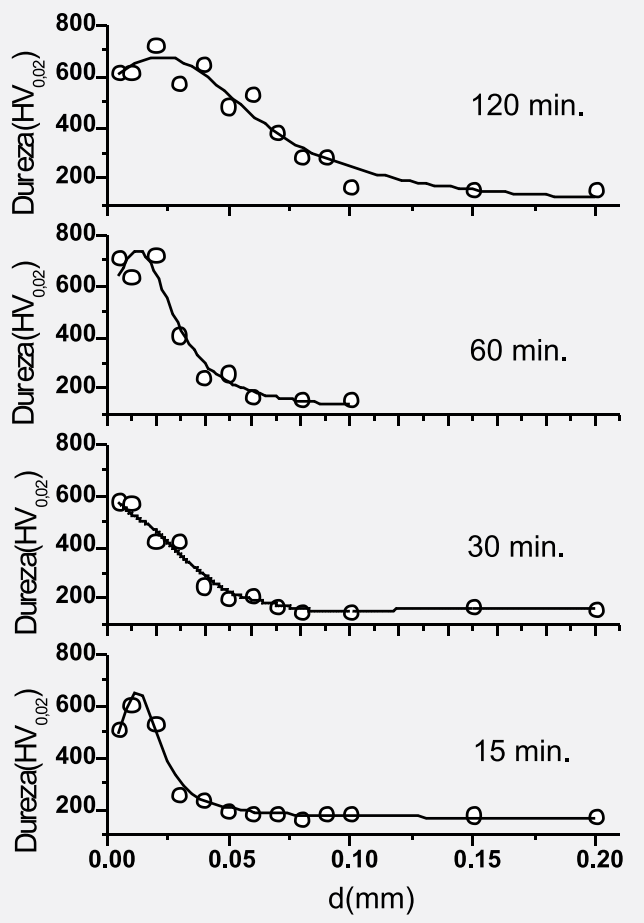

$28 \% \mathrm{Cr}$ 
Con el incremento del contenido de cromo, también disminuye el coeficiente de actividad del nitrógeno en la aleación de FeCr (Gavriljuk e Berns, 1999) y, por tanto, aumenta la cantidad (de nitrógeno) absorbida en la misma. Esto se observa claramente en la Figura 3(b) la cual fue trazada con los mismos datos de la Figura 3(a). Este comportamiento de la aleación de $\mathrm{Fe}$-Cr fue evaluado en estudios bajo consideraciones termodinámicas (Frisk, 1990).

Los perfiles de microdureza para cada composición de la aleación de $\mathrm{Fe}-\mathrm{Cr}$ son mostrados en la Figura 4 para varios tiempos $(15,30,60$ y 120 min.) de absorción de nitrógeno en la descarga luminiscente anormal en atmósfera de nitrógeno a $950^{\circ} \mathrm{C}$. Los valores de la microdureza determinados para la martensita formada en la aleación con 13\% Cr en los tiempos de 15 y 30 min. (Sarmiento, S. A., 2003), están entre los valores reportados en trabajos anteriores para este tipo de acero con nitrógeno (Horovitz et al, 1996). Para tiempos de tratamiento superiores 60 minutos, en esta aleación, y para las demás aleaciones utilizadas (18, 23 y $28 \%$ de cromo), donde se forman precipitados de nitruro de cromo (Sarmiento y Muzart, 2006), se observa un aumento del valor de la microdureza, alcanzando valores característicos en los aceros al cromo con estos precipitados de nitruro de cromo (Chiu et al, 2002)

El valor máximo de la dureza tiene un leve aumento con el contenido de cromo, debido al aumento de la cantidad de precipitados de nitruro de cromo, en las aleaciones con mayor capacidad para absorber nitrogenito de la atmósfera gaseosa. Por otro lado, a pesar del contenido de nitrógeno ser mayor en aleaciones con mayor contenido de cromo, el ancho del perfil de microdureza se ve disminuido con el mismo aumento del contenido de cromo en la aleación.
Esto es debido la disminución de la actividad del nitrógeno, con el incremento del contenido de cromo, como fue discutido en el párrafo anterior.

No se observa incidencia importante de tiempo en el valor máximo de la microdureza para las aleaciones con contenidos de cromo superiores al 13\%, lo que podría indicar que la superficie de la aleación absorbe el nitrógeno en cantidad suficiente para generar las estructuras duras en un tiempo pequeño ( $<15 \mathrm{~min}$ ), a partir del cual todo el nitrógeno adicional pasa hacia el interior de la muestra, por difusión a través de esta estructura dura, alargándola, como se observa en la Figura 4 para cada composición de cromo estudiada. El tiempo de proceso contribuye a aumentar el ancho del perfil e microdureza para cada composición de cromo, como es de esperarse en todo proceso difusional.

\section{Conclusiones}

El valor máximo de la microdureza de una aleación inoxidable de $\mathrm{Fe}-\mathrm{Cr}$, sometida a una descarga luminiscente anormal en atmósfera de nitrógeno, aumenta levemente con el contenido de cromo de la muestra mientras que el perfil de dureza se estrecha, debido al hecho del nitrógeno presentar fuerte tendencia a formar nitruros y mayor dificultad para difundirse, respectivamente, en aleaciones con mayor contenido de cromo.

\section{Bibliografía}

Chapman, B., Glow Discharge Processes, John Wiley and Sons, New York, 1980.

Chiu, L. H., Yang, C. F., Hsieh, W. C., Cheng, A. S., Effect of Contact Pressure on Wear Resistance of AISI H13 Tool Steels with Chromium Nitride and Hard Chromium 
Coatings, Surface and Coatings, V. 154, p. 282-288, 2002.

Feichtinger, H. K., Zeng, XH., Thermodinamik und Verfahrenstechnik der Herstellung von Stickstoff-Stählen, 1991.

Frisk, K., CALPHAD, V.15, p.79, 1991.

Gavriljuk, V. J., Berns, H., High Nitrogen Steels, Springer-Verlag Berlin Heilderberg, 1999.

Hertzman, S., Jarl, M., A Thermodynamic Analysis of the Fe-Cr-N System, Metallurgical Transactions A, V. 18A, p. 1745-1752, 1987.

Horovitz, M. B., Beneduce Neto, F., Garbogin, A., Tschiptschin, A. P., Nitrogen Bearing Martensitic Stainless Steels: Microstructure and Properties, ISIJ International., V. 36, n. 7, p. 840-845, 1996.

Sarmiento, S. A. y Muzart, J. L. R., Disolución del Nitruro cúbico de Cromo en la Descarga Luminiscente Anormal, Revista Colombiana de Física V. 38, No. 1, p. 265-268, 2006.

Sarmiento, S. A. y Muzart, J. L. R., Caracterización Microestructural de Aleaciones de Fe-Cr Sometidas a la Descarga Luminiscente Anormal en Atmósfera de Nitrógeno, Revista Colombiana de Física V. 38, No. 1, p. 261-264, 2006.

Sarmiento, S. A., Introdução de Nitrogênio em Ligas Sinterizadas de Fe-Cr, Submetidas a uma Descarga Luminescente Anormal, Tesis, UFSC, Florianópolis, Brasil, 2003.

Wriedt, H. A., Gokcen, N. A., Nafziger, R. H., The Fe-N (Iron-Nitrogen) System, Bulletin of Alloy Phase Diagrams, V. 45, n. 4, p. 355, 1987.

Zeng, XH, Satir-Kolorz, A., Feichtinger, $\mathrm{H}$. K., HNS'88-High Nitrogen Steels, p. 75, Ins. Met., London, 1989. 